\title{
Green Synthesis of Nano-Sized Sulfur and Its Effect on Plant Growth
}

\author{
Nidá M. Salem ${ }^{1}$, Luma S. Albanna ${ }^{1}$, Akl M. Awwad², Qusay M. Ibrahim ${ }^{2} \&$ Amany O. Abdeen ${ }^{2}$ \\ ${ }^{1}$ Department of Plant Protection, Faculty of Agriculture, The University of Jordan, Amman, Jordan \\ ${ }^{2}$ Department of Nanotechnology, Royal Scientific Society, Amman, Jordan \\ Correspondence: Akl M. Awwad, Department of Nanotechnology, Royal Scientific Society, P.O. Box 1438, \\ Amman 11941, Jordan. Tel: 962-6534-4701. E-mail: akl.awwad@yahoo.com; akl.awwad@rss.jo
}

Received: October 21, 2015 Accepted: November 26, 2015 Online Published: December 15, 2015

doi:10.5539/jas.v8n1p188 URL: http://dx.doi.org/10.5539/jas.v8n1p188

\begin{abstract}
The major objective of this research paper is to synthesis sulfur nanoparticles (SNPs) by green route, which can improve plant's growth and reduce the environmental pathogens. Sulfur nanoparticles (SNPs) were synthesized by a simple green procedure using Melia azedarach leaves aqueous extract and citric acid. The leaves of Melia azedarach aqueous extract act as a capping and stabilizing agent in the formation of sulfur nanoparticles. The synthesized sulfur nanoparticles were characterized by X-ray diffraction (XRD), scanning electron microscopy (SEM), and Fourier transform infrared spectroscopy (FT-IR). The average particles diameter size was found to be $20 \pm 4 \mathrm{~nm}$. Analysis showed that the nanoparticles are crystalline in nature, with spherical shape. A preliminary field study was conducted to evaluate growth and development of Cucurbita pepo in response to sulfur nanoparticles.
\end{abstract}

Keywords: green synthesis, sulfur nanoparticles, Melia azedarach leaves, plant's growth, characterization

\section{Introduction}

Nanotechnology has gained intense attention in the recent years due to its wide application in diverse areas like medicine, catalysis, energy and materials. Particularly nanoparticles with small size to large surface area have potential medical as well as industrial applications. Researchers make significant efforts towards the synthesis of nanoparticles by various means, including physical, chemical and biological methods. Green methods for synthesizing nanoparticles with plant extracts are advantageous over physical, chemical and microbial methods as it is simple, convenient, environment friendly, and requires less reaction time. Sulfur has a wide range of applications in different agriculture area. Sulfur can be used as fungicide against many plant diseases such as the apple scab disease in the cold conditions, also Sulfur used in the culture of grapes, vegetables, strawberry and many cultivated plants. However, sulfur can be considered as a high efficiency pesticide that used in agriculture where it has good effect against a wide range of powdery mildew diseases as well as black spot. Sulfur nanoparticles have many advantages over micro-sulfur for their peculiar quantum size properties and high surface areas. Recently, great effort has been focused on finding approaches for synthesis sulfur nanoparticles (SNPs) with well-defined shapes and nano-sizes. Among these, sulfur nanoparticles synthesized from $\mathrm{H}_{2} \mathrm{~S}$ gas by using biodegradable iron chelates catalyst in reverse microemulsion technique (Deshpande et al., 2008; Guo et al., 2006), an electrochemical method using thiosulfate ion (Shamsipur et al., 2011), water-in-oil microemulsion system using cyclohexane as oil phase, butanol as co-surfactant, nonionic surfactant Triton X-100 and sodium polysulfide (Soleimani et al., 2013), an aqueous solution of potassium polysulfide and various organic and inorganic acids (Massalimov et al., 2012), sublimed sulfur in ethanol using ultrasonic technique (Xie et al., 2009), dissolving sublimed sulfur in a green solvent PEG-400 (Xie et al., 2012), aqueous surfactant (Chaudhuri and Paria, 2012), supersaturated solvent method (Wu et al., 2008), heating sulfur powder with polyethylene glycol PEG-600 (Meenatchi et al., 2015), and eggshell membrane as natural biomaterial (cheng et al., 2011). These methods have many disadvantages due to the difficulty of scale up of the process, separation and purification of nanoparticles from the microemulsion (oil, surfactant, co-surfactant and aqueous phase), and consuming huge amount of surfactant. In previous work, sulfur nanoparticles synthesized by green method from sodium thiosulfate in presence of Albizia julibrissin fruits extract (Awwad et al., 2015). As continuation of our previous work, we report here for the first time a novel, rapid, cost-effective and environmentally biosynthesis of sulfur nanoparticles using Melia azedarach leaves extract at room temperature. The major objectives of this research is 
to synthesis sulfur nanoparticles by an environmentally green method, which can improve plant's growth and reduce the environmental risks as those caused by pathogenic organisms.

\section{Materials and Methods}

\subsection{Materials}

Sodium thiosulfate pentaahydrate $\left(\mathrm{Na}_{2} \mathrm{~S}_{2} \mathrm{O}_{3} \cdot 5 \mathrm{H}_{2} \mathrm{O}, 99.8 \%\right)$ and anhydrous citric acid $\left(\mathrm{C}_{6} \mathrm{H}_{8} \mathrm{O}_{7}, 99 \%\right)$ were obtained from Merck, Darmstadt, Germany and used as received without any further purification. Sterile distilled water was used with conductivity $1 \mu \mathrm{S} / \mathrm{cm}$.

\subsection{Preparation of Melia Azedarach Leaves Extract}

Fresh leaves of Melia azedarach were collected from the campus of Royal Scientific Society, Jordan. Fresh leaves were washed under running tap water to remove any debris and dust attached to the leaves and subsequently with distilled water 3-4 times. After washing the leaves were separated by cutting them into small pieces and air dried for four weeks at room temperature. The dried leaves were finely powdered through grinding using Restch RM 100 grinder. The extract was prepared by taking $20 \mathrm{~g}$ of powdered leaves and boiled in $500 \mathrm{ml}$ glass beaker along with $400 \mathrm{ml}$ of sterile distilled water for 10 minutes. The aqueous extract of leaves was separated by filtration with Whatman No. 1 filter paper and then centrifuged at $1200 \mathrm{rpm}$ for 5 minutes to remove heavy biomaterials. The filtrate was collected and stored at room temperature. This gave the final leaves extract which was used for further experimental procedure.

\subsection{Synthesis of Sulfur Nanoparticles}

In a typical reaction synthesis, sulfur nanoparticles (SNPs) synthesized as follows: An appropriate amount of sodium thiosulfate pentahydrate $\left(\mathrm{Na}_{2} \mathrm{~S}_{2} \mathrm{O}_{3} .5 \mathrm{H}_{2} \mathrm{O}\right)$ was dissolved in $100 \mathrm{ml}$ of Melia azedarach leaves extract under mild stirring for 5 minutes at room temperature and then diluted with deionized distilled water to $500 \mathrm{ml}$. Afterwards citric acid $(0.5 \mathrm{~g} / \mathrm{ml})$ was added drop by drop under stirring for allowing the sulfur precipitations uniformly. The suspended sulfur particles obtained were separated by centrifugation at $1000 \mathrm{rpm} / \mathrm{min}$ for 5 minutes and then repeatedly washed with sterile distilled water to remove any biological materials. Sulfur nanoparticles after purification were dried in a vacuum at $80{ }^{\circ} \mathrm{C}$ for $6 \mathrm{~h}$. The product was light yellow powder used for SEM, XRD, and FTIR analysis.

\subsection{Characterization Techniques}

Crystalline sulfur nanoparticles were examined by X-ray diffractometer (XRD-6000 Shimadzu, Japan) equipped with $\mathrm{Cu} \mathrm{K} \alpha$ radiation source using $\mathrm{Ni}$ as filter and at a setting of $30 \mathrm{kV} / 30 \mathrm{~mA}$. All XRD data were collected under the same experimental conditions, in the angular range $3^{\circ} \leq 2 \theta \leq 60^{\circ}$. Fourier transmission infrared (FT-IR) spectra for Melia azedarach leaves extract and sulfur nanoparticles were obtained in the range $4000-400 \mathrm{~cm}^{-1}$ by IR-Prestige-21, FT-IR spectrophotometer (Shimaduz, Japan) using $\mathrm{KBr}$ pellet method. Scanning electron microscopy (SEM) analysis of sulfur nanoparticles was done using Quanta FEI 450 SEM machine.

\subsection{Plant Growth and Sulfur Nanoparticles}

Experiments were conducted in $10 \mathrm{~m} \times 10 \mathrm{~m}$ greenhouse compartments, which were part of the Department of Plant Protection, Faculty of Agriculture, the University of Jordan. In all experiments, the indeterminately growing round Cucurbita pepo was used. After 10 days sowing in trays filled with a commercial potting soil, plants were picked out and transferred to a field at the Royal Scientific Society, where the grown in soil of the field. The response of $C$. pepo to sulfur nanoparticles was studied through field experiments conducted during April-May 2015. Sulfur nanoparticles (SNPs) were applied to the field soil $100 \mathrm{ppm}-600 \mathrm{ppm}$ doses. Plant height was measured from bottom to top of C. pepo. Stem diameter was measured from base, middle and top portion and average was taken. Number of branches and leaves were counted.

\section{Results and Discussion}

\subsection{X-Ray Diffraction Analysis (XRD)}

The XRD analysis of the synthesized sulfur nanoparticles is illustrated in Figure 1 . The $2 \theta$ peaks at $15.26^{\circ}$, $22.82^{\circ}, 25.54^{\circ}, 27.58^{\circ}, 28.44^{\circ}, 31.12^{\circ}, 33.96^{\circ}, 36.78^{\circ}, 42.48^{\circ}, 47.52^{\circ}$, and $51.06^{\circ}$ are attributed to the crystal planes of sulfur. The sulfur nanoparticles are well-crystalline and the relative intensity of the diffraction peaks match well with the standard monoclinic phase sulfur diffraction pattern (JCPDS 08-0247). The average particle sizes of the synthesized sulfur nanoparticles were calculated using Debye-Scherrer formula (Klug \& Alexander, 1954):

$$
\mathrm{D}=\mathrm{K} \cdot \lambda / \beta \cdot \cos \theta
$$


Where $\mathrm{D}$ is the mean diameter of nanoparticles, $\beta$ is the full width at half-maximum value of XRD diffraction lines, $\lambda$ is the wavelength of X-ray radiation source $0.15405 \mathrm{~nm}, \theta$ is the half diffraction angle-Bragg angle and $\mathrm{K}$ is the Scherrer constant with value from 0.9 to 1 . The unassigned peaks in XRD pattern are thought to be related to crystalline and amorphous organic phases of Melia azedarach leaves extract. The crystalline size of sulfur nanoparticles calculated from Scherrer equation was about $20 \mathrm{~nm}$.

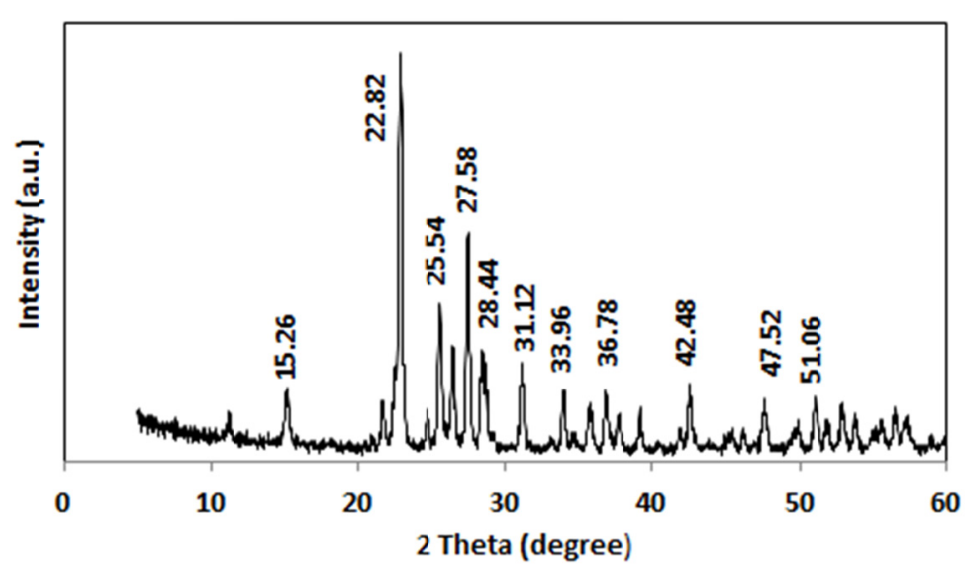

Figure 1. XRD pattern of the synthesized sulfur nanoparticles

\subsection{Fourier Transmission Infrared Analysis (FT-IR)}

FT-IR analysis was carried out to identify the possible biomolecules responsible for the capping and stabilization of sulfur nanoparticles synthesized by Melia azedarach leaves extract. The extract display a number of absorption peaks, reflecting their complex nature, Figure 2. FT-IR spectrum of Melia azedarach leaves extract display strong absorption bands at $3568 \mathrm{~cm}^{-1}-3248 \mathrm{~cm}^{-1}$ could be ascribed to the stretching absorption band of amino (-NH) and hydroxyl (-OH) stretching H-bonded alcohols and phenols. The strong absorption peaks at $2924 \mathrm{~cm}^{-1}$ and $2854 \mathrm{~cm}^{-1}$ could be assigned to the asymmetric and symmetric stretching of $-\mathrm{CH},-\mathrm{CH}_{2}$ and $-\mathrm{CH}_{3}$ functional groups of aliphatic compounds in the plant.

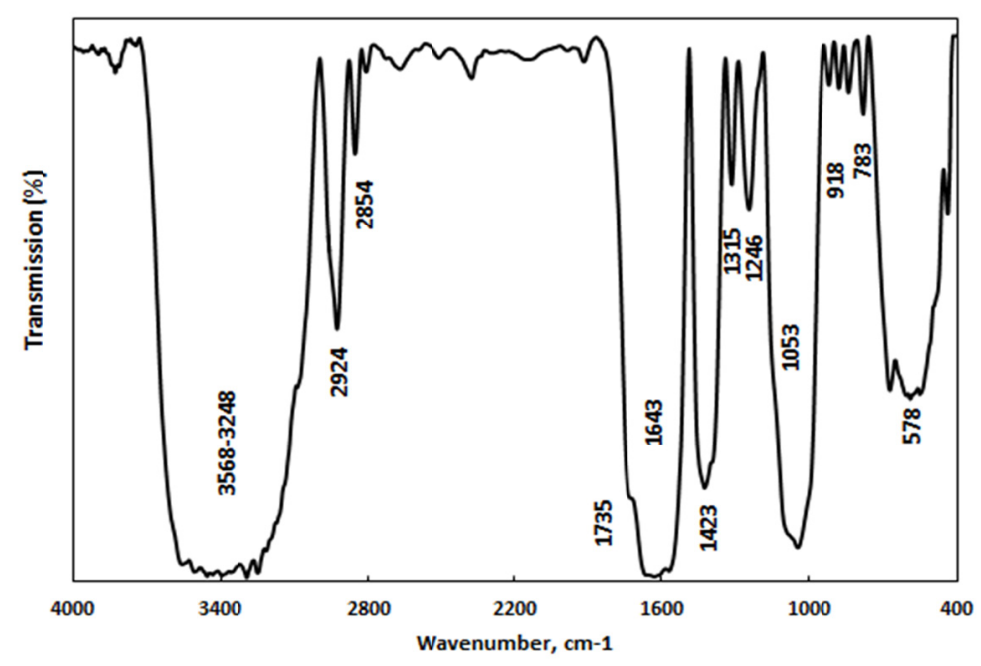

Figure 2. FT-IR spectrum of Melia azedarach leaves extract

The shoulder peak at $1735 \mathrm{~cm}^{-1}$ corresponds to stretching to carboxyl group. The bands at $1643 \mathrm{~cm}^{-1}$ is characteristic of amide carbonyl group in amide I and amide II. The amide band I assigned to the stretch mode of the carbonyl group coupled to the amide linkage while the amide II band arises as a result of the N-H stretching modes of vibration in the amide linkage. The peak at $1423 \mathrm{~cm}^{-1}$ is assigned to the methylene scissoring 
vibrations from the proteins. C-N stretch of aromatic amines and carboxylic acids gives rise to bands at 1315 $\mathrm{cm}^{-1}$. The peak at $1246 \mathrm{~cm}^{-1}$ is due to C-O vibrations of alcohols, phenols and $\mathrm{C}-\mathrm{N}$ stretching vibrations of amine. The band at $1053 \mathrm{~cm}^{-1}$ assigned to the C-O stretching vibrations of alcohols $\mathrm{C}-\mathrm{N}$ stretching vibrations of amine. The peaks at $918 \mathrm{~cm}^{-1}, 783 \mathrm{~cm}^{-1}, 675 \mathrm{~cm}^{-1}$ and $578 \mathrm{~cm}^{-1}$ can be assigned to bending modes of aromatic compounds.

FT-IR spectra of SNPs, Figure 3 indicate a new chemistry linkage on the surface of sulfur nanoparticles. This suggests that Melia azedarach leaves extract can bind to sulfur nanoparticles through carbonyl of the amino acid residues in the protein of the extracts, therefore acting as stabilizer and dispersing agent for synthesized sulfur nanoparticles and prevent agglomeration of sulfur nanoparticles. All the characteristic peaks of Melia azedarach leaves extract were observed in FT-IR spectra of sulfur nanoparticles. No significant new peak appeared, indicating there was no chemical reaction but physical cross-linking between peel extract and sulfur nanoparticles. FT-IR spectra of sulfur nanoparticles show a strong and sharp peak at $466 \mathrm{~cm}^{-1}$. The results of the phytochemical analysis of Melia azedarach leaves aqueous extract showed that the polyphenols, alkaloids, tannins, and amino acids were found to be present in afore mentioned leaves extract (Harborne, 1973). The plant leaves screened for phytochemical constituents seemed to have the potential to act as a source of capping and also to stabilize the nanoparticles as a result of the presence of various biomaterials that are vital for dispersing, capping, and stabilization.

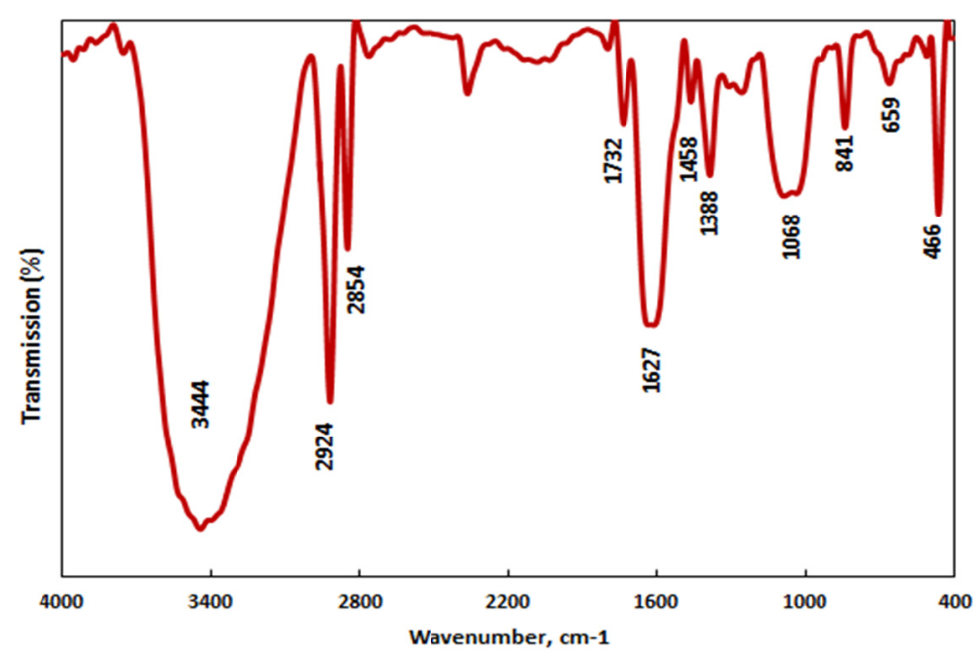

Figure 3. FT-IR spectrum of synthesized sulfur nanoparticles

\subsection{Scanning Electron Microscopy Analysis (SEM)}

The suspended sulfur nanoparticles in sterile distilled water were used for scanning electron microscopy. SEM carried out by fabricating a drop of suspension onto a clean electric stubs and allowing water to completely evaporate. The SEM images of the sulfur nanoparticles synthesized by Melia azedarach leaves extract show all the particles are homogeneously dispersed and ranges approximately from 5-80 nm. The shape of the sulfur nanoparticles is spherical with few exceptional as ellipsoidal. The larger sulfur nanoparticles may be due to the aggregation of the smaller ones, due to the SEM measurements. It was found the increasing the amount of plant extract in the reaction mixture leads to decrease the particles size of sulfur nanoparticles and their agglomeration tendencies.

\subsection{Plant Growth}

The importance of Cucurbita pepo as a vegetable crop has been long recognized worldwide due to its high nutritional and economic value. In Jordan the fruits of Cucurbita pepo are cooked to prepare different dishes. It has a wide range of genetic variability, both in vegetative and reproductive characteristics . The yield and quality of fresh Cucurbita pepo are affected by both genetic factor and growing condition. In this work, a preliminary study on the effect of the synthesized sulfur nanoparticles on Cucurbita pepo growth investigated. Cucurbita Pepo growth in soil treated with sulfur nanoparticles compared with non-treated (control) was positively affected. SNPs treatment of the soil increased the number of leaves, number of branches, height per plant, stem diameter, 
and healthy plant. These results, for the first time, demonstrate the successful use of sulfur nanoparticles (100 $400 \mathrm{ppm}$ ) in enhancing C. pepo growth under field conditions. Energy-dispersive X-ray fluoresence spectroscopy (EDS) analysis to the roots and stems of Cucurbita pepo planted in soil treated with sulfur nanoparticles show the presence of sulfur nanoparticles, which enhances the growth, Figures 4 and 5.

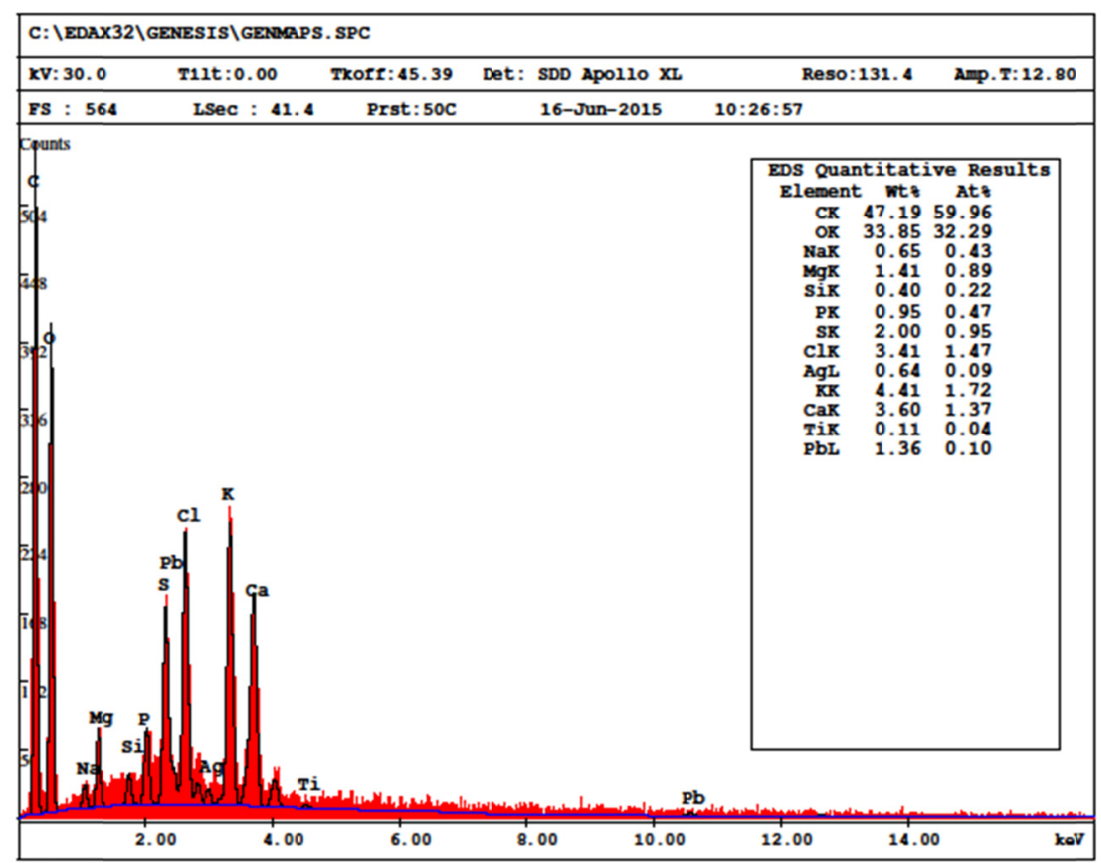

Figure 4. Energy-dispersive X-ray fluorescence spectroscopy spectrum of root of Cucurbita pepo

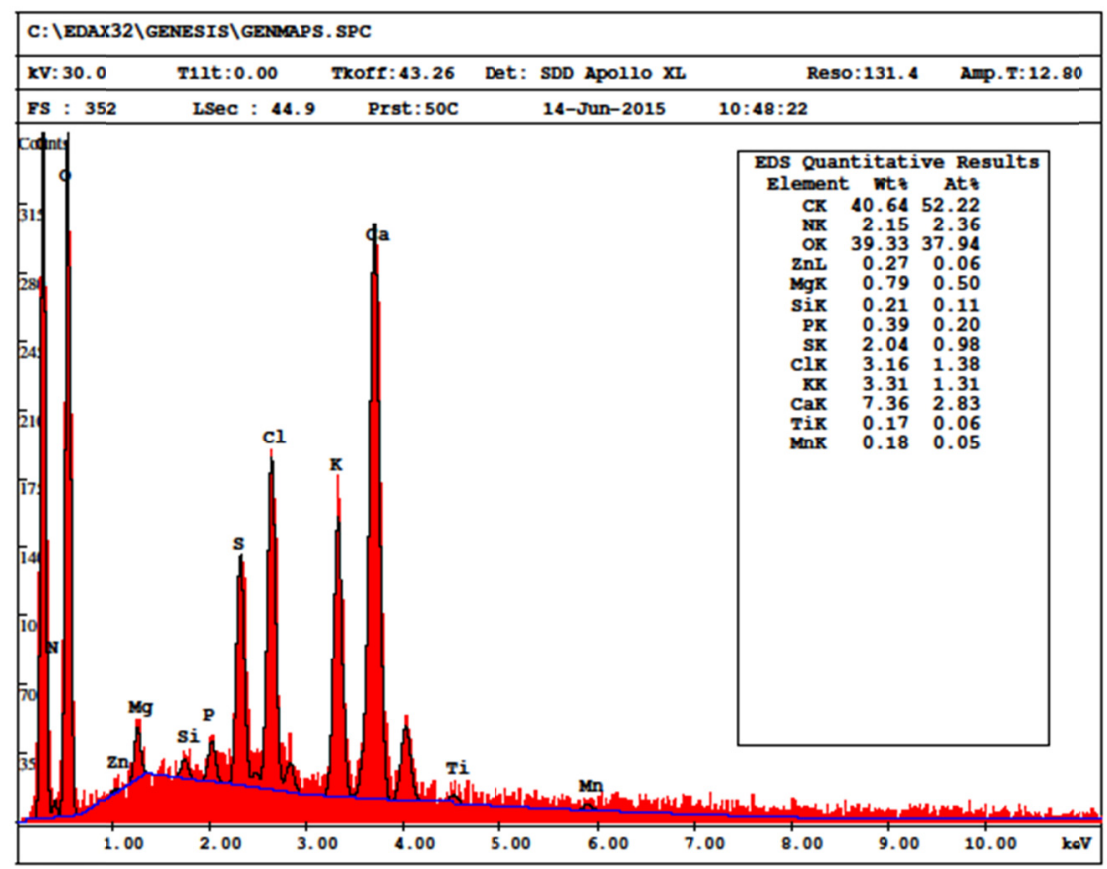

Figure 5. Energy-dispersive X-ray fluorescence spectroscopy spectrum of stem of Cucurbita pepo

The effect of of sulfur nanoparticles on root and shoot lengths of C. pepo are shown in Figure 6. It was observed that an increase in SNPs concentration, the shoot and root lengths incresased. After certain SNPs concentration, $600 \mathrm{ppm}$, it was found that SNPs has no effect on shoot and root growth. These preliminary results indicated that 
SNPs suppress crop disease and subsequently enhance the Cucurbita pepo growth. This enhancement suggests that a result of the reduction of the presence of pathogenic organisms in the soil and the potential nutritional value of SNPs through producing sulfur organic compounds in the plant. A detailed study is now carried out to give complete picture on the effect of SNPs on the soil and plant growth.

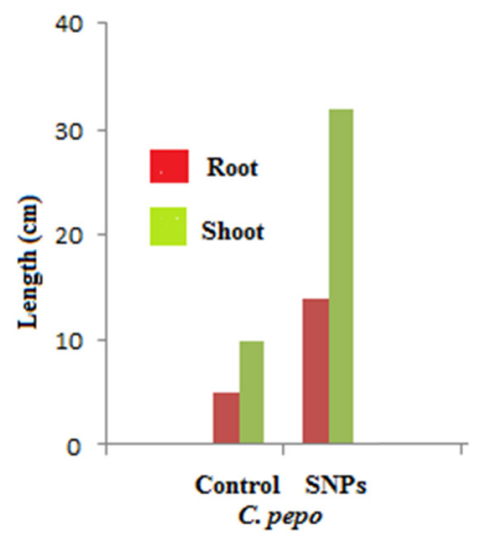

Figure 6. Length $(\mathrm{cm})$ of root and shoot $C$. pepo plant as affected by soil tratment by sulfur nanoparticles

\section{Conclusion}

A novel approach for green synthesis of sulfur nanoparticles by using Melia azedarach leaves extract in one-pot liquid phase reaction at ambient temperature and atmospheric pressure has been reported for first time. The described approach gives highly crystalline pure sulfur nanoparticles with uniform shape and average particle size of 5-80 $\mathrm{nm}$. The concentrations of Melia azedarach leaves extract and sodium thiosulfate ions are playing an important role in the green synthesis of sulfur nanoparticles. XRD, SEM and FT-IR support the formation and stability of the biosynthesized SNPs. Thus it can be concluded that plant extracts can be used as simple, low-cost and environmentally friendly biomaterial acting as stabilizing and dispersing agent in synthesis of SNPs. Preliminary results indicated that SNPs suppress crop disease and subsequently enhance the Cucurbita pepo growth. This enhancement suggests that a result of the reduction of the presence of pathogenic organisms in the soil and the potential nutritional value of SNPs through producing sulfur organic compounds in the plant.

\section{Acknowledgements}

The authors thank financial supports from Scientific Research Support Fund (SRSF/No. Agr/2/13/2013/7-2015), Ministry of Higher Education and Scientific Research, Jordan.

\section{References}

Awwad, A. M., Salem, N. M., \& Abdeen, A. O. (2015). Novel approach for synthesis sulfur (S-NPs) nanoparticles using Albizias julibrissin fruits extract. Adv. Mater. Lett., 6, $432-435$. http://dx.doi.org/10.5185/amlett.2015.5792

Chaudhuri, R. G., \& Paria, S. J. (2010). Synthesis of sulfur nanoparticles in aqueous surfactant solutions. $J$. Colloid and Interface Sci., 343, 439-446. http://dx.doi.org/ 10.1016/j. jcis.2009.12.004

Cheng, X., Cheng, K., Liu, J., \& Sun, X. (2011). Synthesis and characterizations of nanoparticle sulfur using eggshell membrane as template. Mater. Sci. Forum, 675-677, $279-282$. http://dx.doi.org/10.4028/www.scientific.net/MSF.675-677.279

Deshpande, A. S., Khomane, R. B., Vaidya, B. K., Joshi, R. M., Harie, A. S., \& Kulkarni, B. D. (2008). Sulfur nanoparticles synthesis and characterization from $\mathrm{H}_{2} \mathrm{~S}$ gas, using novel biodegradable iron chelates in W/O microemulsion. Nanoscale Res. Lett., 3, 221-229. http://dx.doi.org/ 10.1007/s11671-008-9140-6

Guo, Y., Zhao, J., Yang, S., Yu, K., Wang, Z., \& Zhang, H. (2006). Preparation and characterization of monoclinic sulfur nanoparticles by water-in-oil microemulsions technique. Powder Technology, 162, 83-86. http://dx.doi.org/ 10.1016/j.powtec.2005.12.012

Harborne, J. B. (1973). Phytochemical methods. London Chapman and Hall, Ltd.

Klug, H. P., \& Alexander, L. E. (1954). X-ray diffraction procedure for polycrystalline and amorphous materials. 
Wiley, New York.

Massalimov, I. A., Shainurova, A. R., Khusainov, A. M., \& Mustafin, A. G. (2012). Production of sulfur nanoparticles from aqueous solution of potassium polysulfide. Russian J. Appl. Chem., 85, 1832-1837. http://dx.doi.org/ 10.1134/S1070427212120075

Meenatchi, B., \& Renuga, V. (2015). Protic ionic liquids assisted synthesis and characterization of sulfur nanoparticles and $\mathrm{CdS}$ and $\mathrm{ZnS}$ nanomaterials. Chemical Science Transactions, 4, 577-587. http://dx.doi.org/10.7598/cst2015.1028

Shamsipur, M., Poourmortazavi, S. M., Roushani, M., Kohsar, I., \& Hajimirsadeghi, S. (2011). Novel approach for electrochemical preparation of sulfur nanoparticles. Microchimica Acta, 173, 445-451. http://dx.doi.org/10.1007/s00604-011-0581-8

Soleimani, M., Aflatouni, F., \& Khani, A. A. (2013). New and simple method for sulfur nanoparticles synthesis. Colloid Journal, 75, 112-116. http://dx.doi.org/ 10.1134/S1061933X12060142

Wu, H., Wang, W., Yin, H., Zhang, D., Jiang, T., Zhang, R., \& Liu, Y. (2008). Preparation of sulfur sheets by supersaturated solvent method in the presence of organic modifiers. Mater. Lett., 62, 1996-1998. http://dx.doi.org/ 10.1016/j.matlet.2007.11.001

Xie, X.-Y., Li, L.-Y., Zheng, P.-S., Zheng, W.-J., Bai, Y., Cheng, T.-F., \& Liu, A. (2012). Facile synthesis, spectral properties and formation mechanism of sulfur nanorods in PEG-200. Mater. Res. Bulletin, 47, 3665-3669. http://dx.doi.org/ 10.1016/j.matteresbull.2012.06.043

Xie, X.-Y., Zheng, W.-J., Bai, Y., \& Liu, J. (2009). Cystine modified nano-sulfur and its spectral properties. Mater. Lett., 63, 1374-1376. http://dx.doi.org/ 10.1016/j.matlet.2008.12.049

\section{Copyrights}

Copyright for this article is retained by the author(s), with first publication rights granted to the journal.

This is an open-access article distributed under the terms and conditions of the Creative Commons Attribution license (http://creativecommons.org/licenses/by/3.0/). 